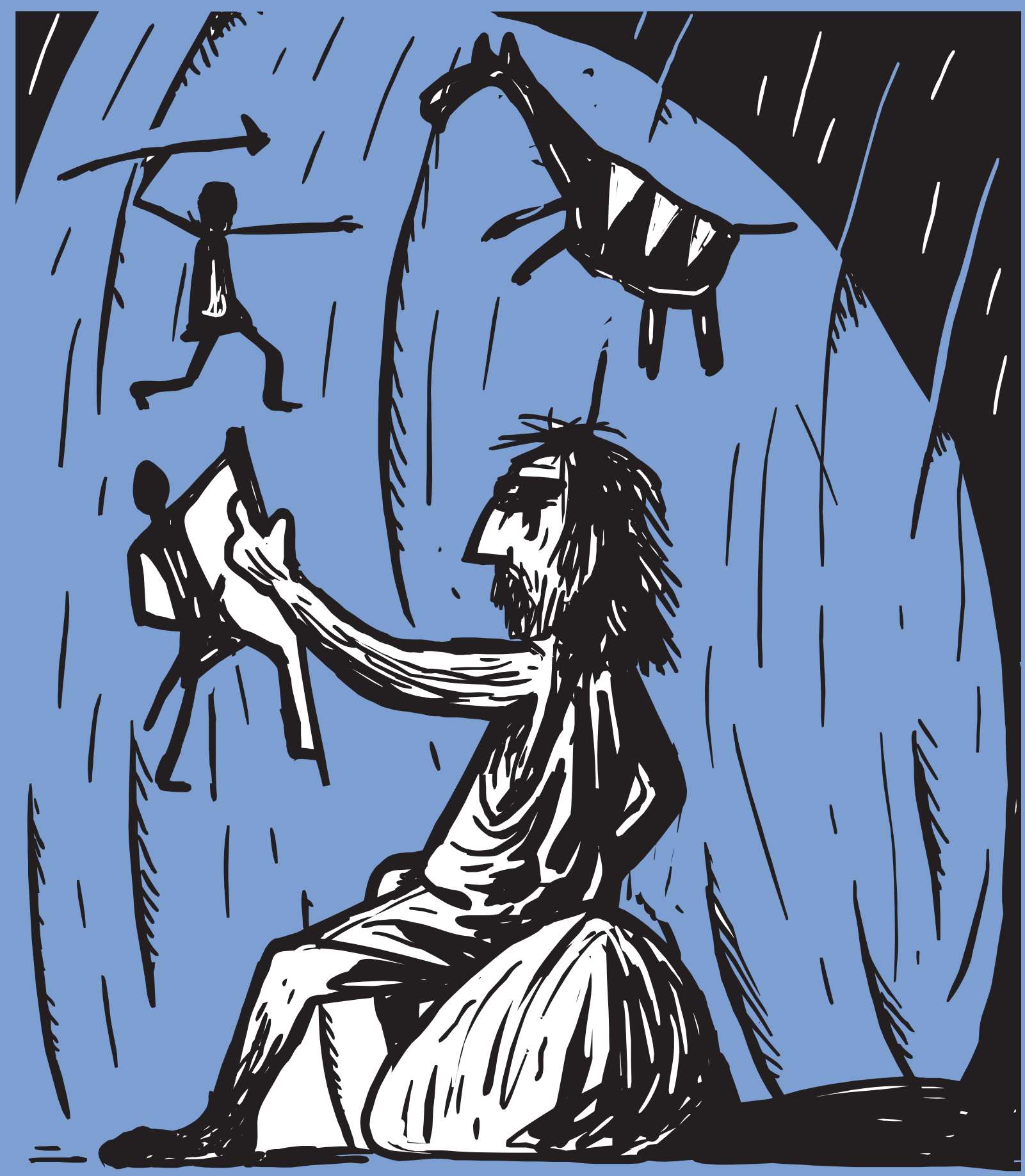




\section{La posible contribución de Marshall McLuhan y la ecología de los medios al desarrollo y comprensión de las \\ comunicaciones estratégicas}

Octavio Islas

- Doctor en Ciencias Sociales por la Universidad La Salle

- Maestro en Comunicación y Desarrollo por la Universidad Iberoamericana

- Director de Proyecto Internet de la Cátedra de Comunicación Estratégica y Cibercultura del Tecnológico de Monterrey, Campus Estado de México

- Miembro del Sistema Nacional de Investigadores de México

- Coordinador del grupo de investigación dedicado al estudio de Internet y la Sociedad de la Información en la coordinación de los consejos editoriales de Razón y Palabra [http://www.razonypalabra.org.mx] y Revista Mexicana de Comunicación

- octavio.islas@itesm.mx 
Resumo

O propósito deste texto é excursionar pelas possíveis contribuições do pensamento de Marshall McLuhan e da chamada ecologia dos meios (Media Ecology), na compreensão das Comunicações Institucionais estratégicas. Os fundamentos para compreender a era digital - afirma acertadamente o pesquisador americano Paul Levinson - estavam na estante dos livros de McLuhan.

PALAVRAS-CHAVE: MCLUHAN • ECOLOGIA dOS MEIOS - COMUNICAÇÕES ESTRATÉGICAS

\section{Abstract}

The purpose of this text is to check the possible contributions of the thought of Marshall McLuhan and of the Media Ecology in the understanding of the strategic Institutional Communications. The basics to understand the digital era - correctly affirms the American outstanding researcher Paul Levinson -, were in the shelf of the books of McLuhan.

KEY WORDS: MCLUHAN • MEDIA ECOLOGY - STRATEGIC COMMUNICATIONS

\section{Resumen}

El propósito de este texto es incursionar en las posibles contribuciones del pensamiento de Marshall McLuhan y de la llamada ecología de los medios (Media Ecology), en la comprensión de las comunicaciones institucionales estratégicas. Los fundamentos para comprender la era digital - afirma atinadamente el destacado investigador estadounidense Paul Levinson -, estaban en el estante de los libros de McLuhan.

PALABRAS CLAVE: MCLUHAN - ECOLOGía de medios - COMUNICACIONES ESTRATÉGICAS 
"Él fue Internet en la década de 1960. Finalmente, el mundo se ha puesto a su altura".

Robert Logan

\section{La Galaxia McLuhan}

$\mathrm{E}$ ste año se cumplirá el cuadragésimo segundo aniversario de la primera edición de Understanding media: the extensions of man (Comprender a los medios de comunicación: las extensiones del hombre), tercer libro de Marshall McLuhan, ${ }^{1}$ cuya primera edición en inglés fue publicada por la editorial McGraw-Hill Book Co., en 1964. Cinco años después Editorial Diana publicó la primera edición en castellano (1969), y en 2003 la editorial Ginko Press sacó a la venta la "versión crítica" del libro más importante de Marshall McLuhan, aunque no precisamente su best seller, el cual ha sido traducido a más de 20 idiomas.

La primera edición de Comprender a los medios dio amplia y positiva notoriedad al célebre pensador canadiense, particularmente en los Estados Unidos. La aguda metáfora el medio es el mensaje, admite ser considerada como una de las tesis axiales en el desarrollo de las Ciencias de la Comunicación.

"McLuhan también introdujo la noción de aldea global, que describió como un mundo conectado gracias a los medios electrónicos. Afirmó que las transmisiones vía satélite y otros transmisores (el Sputnik habia realizado una órbita completa alrededor de la Tierra en 1957 y la convirtió en el centro de un entorno dominado por los medios) habian hecho que la sociedad dejara de ser el mundo mecánico, objetivo, no implicado y visual de la imprenta para convertirse en un mundo electrónico que era inmerso, implicado, inmediato y acústico" (HORROCKS, 2004: 12).

El pensamiento de McLuhan además representa la columna vertebral de una de las más importantes escuelas de pensadores de la Comunicación: la Media Ecology (Ecología de los Medios), la cual, de acuerdo con Lance Strate, destacado investigador de la Universidad de Fordham, Nueva York, y presidente de la Media Ecology Association (MEA), también es conocida como Escuela Norteamericana de Comunicación, Escuela de Toronto o Mediología (STRATE, 2004: 5). ${ }^{2}$

1 (Publicado no Brasil pela Cultrix com o título Os meios de Comunicação como extensões do homem, 1999). En 1951 Marshall McLuhan publicó su primer libro: The mechanical bride: folklore of industrial man. New York, Vanguard Press. En 1962 McLuhan publicó su segundo libro: the Gutemberg Galaxy: the making of typographic man (University of Toronto Press).

2 Sobre la contribución de Marshall McLuhan en la construcción y el desarrollo de la escuela dedicada al estudio de la ecología de los medios, recomendamos consultar: CASEY MAN: The intellectual roots of media ecology. En The New Jersey Journal of Communication. Vol. 8. Número 1. Primavera de 2000, p.1-8. 
Neil Postman, quien fue destacado catedrático del Departamento de Cultura y Comunicación de la Universidad de Nueva York, precisamente propuso el nombre de Media Ecology para designar la corriente teórica que recupera las principales tesis de Marshall McLuhan, en la cual, además de McLuhan y del propio Neil Postman, convergen pensadores de la talla de Joshua Meyrowitz, Edmund Carpenter, Elizabeth Eisenstein, James Carey, Walter Ong, Lewis Mumford, Harold Innis, James Morrison, Paul Ryan, Paul Levinson, Lance Strate, Eric Havelock, Susan Sontag y, por supuesto, Eric McLuhan - uno de los seis hijos de Marshall.

De acuerdo con Neil Postman: "la ecología de los medios estudia la forma como los medios de comunicación afectan la percepción humana, la comprensión, los sentimientos y los valores, y como nuestra interacción con los medios facilita o impide nuestras oportunidades de supervivencia”. ${ }^{3}$ La ecología de medios no sólo nos permite comprender los efectos que introducen los medios de comunicación en las sociedades, también nos permite entender cómo los medios institucionales inciden en la dinámica cultural de las organizaciones.

Marshall McLuhan consideraba al telégrafo como la hormona social que dio inicio a la velocidad instantánea en el movimiento de la información. ${ }^{4}$ Los paralelismos que son posibles advertir entre la introducción del telégrafo y el advenimiento de Internet, definitivamente resultan sorprendentes. Es posible comprender la Internet como remediación del telégrafo. De acuerdo con McLuhan, el telégrafo introdujo la era de la angustia. Todos los medios - y por supuesto, todas las tecnologías - admiten ser entendidas como prolongaciones del hombre. Cada tecnología altera la velocidad con la cual el hombre desarrolla determinadas prácticas culturales. La aceleración tecnológica naturalmente viene acompañada de periodos de incertidumbre y tiempos de adaptación a las nuevas circunstancias. Con el telégrafo, afirma McLuhan: "el hombre había iniciado una exteriorización o prolongación de su sistema nervioso central, que actualmente, con las emisiones vía satélite, se acerca a una prolongación de la conciencia" (McLUHAN, 1996: 308).

Bill Gates, en su reciente libro: Business @ the speed of thought. Using a digital nervous system ${ }^{5}$ (Los negocios a la velocidad del pensamiento. Utilizando un sistema nervioso digital), recuperó la referida tesis de McLuhan - la prolongación del sistema nervioso central - para afirmar que los sistemas intranet, extranet e Internet admiten ser consi-

3 Véase: http://www.media-ecology.org Fecha de consulta: 1 de febrero, 2006.

4 Véase el capítulo 25 de Comprender a los medios. Las extensiones del hombre. "El telégrafo. La hormona social".

5 Publicado no Brasil sob o título $A$ empresa na velocidade do pensamento, Companhia das Letras, 1999. (N. E.) 
derados como sistemas nerviosos digitales de las organizaciones del nuevo milenio. Gates además sostiene que si la calidad y la reingeniería fueron paradigmas de competitividad de las organizaciones durante las décadas de 1980 y 1990, la velocidad sería considerada como paradigma de eficiencia organizacional en las organizaciones del nuevo milenio.

"If the 1980s were about quality and the 1990s were about reengineering, then the 2000s will be about velocity. About how quickly the nature of business will change. About how quickly business itself will be transacted. About how information access will alter the lifestyle of consumers and their expectations of business" (GATES, 2000: XIII).

Las nuevas condiciones de competencia del mundo globalizado han erradicado la sensación de relativa seguridad que antaño conferían las fronteras y los aranceles a las economías de los países en vías de desarrollo. Hoy la velocidad en el movimiento de la información repercute en la competitividad misma de las organizaciones. La supervivencia de éstas en buena medida dependerá de su capacidad para comprender rápidamente su entorno, respondiendo de forma eficiente a las exigencias de sus principales audiencias. La efectiva gestión del conocimiento hoy establece la diferencia empresarial. A mayor calidad y pertinencia de la información que circule a través de los sistemas nerviosos digitales, mayores probabilidades de reducir incertidumbre, optando por las mejores decisiones posibles.

\section{La importancia de la comunicación estratégica en el nuevo pensamiento administrativo}

Previamente al formidable boom de Internet y de las comunicaciones digitales extendidas, dos grandes paradigmas del pensamiento administrativo influían decisivamen-

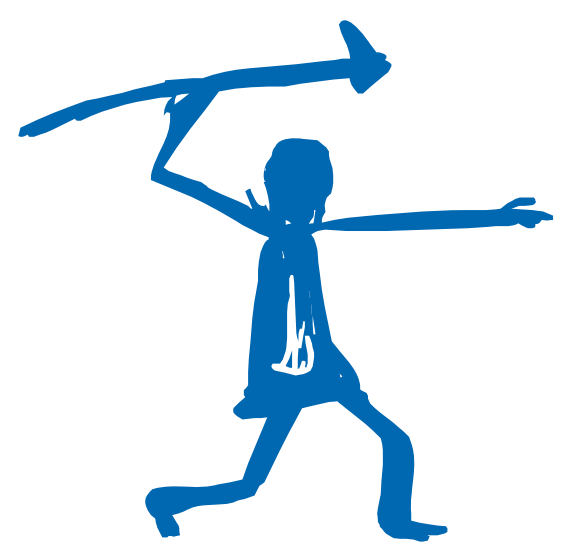


LA POSIBLE CONTRIBUCIÓN DE MARSHALL MCLUHAN Y LA ECOLOGÍA DE LOS MEDIOS AL DESARROLLO Y COMPRENSIÓN DE LAS COMUNICACIONES ESTRATÉGICAS • OCTAVIO ISLAS

te en la gestión empresarial: la dirección por objetivos y el desarrollo organizativo. En ambos paradigmas la Comunicación era considerada asunto periférico. En 1981, Thomas Peters y Robert Waterman publicaron En búsqueda de la excelencia, obra que admite ser considerada auténtico parte aguas en el desarrollo del pensamiento administrativo. Los referidos autores analizaron sesenta y dos de las más importantes empresas de los Estados Unidos, partiendo de la hipótesis de que el organigrama de las empresas no incide en su éxito empresarial. Peters y Waterman consiguieron aislar un conjunto de valores intangibles (invisible assets), los cuales consideraron como factores determinantes de la excelencia. De acuerdo con Hiroyuki Itami, citado por el destacado investigador español Justo Villafañe:

"Los activos invisibles son la fuente real de la competitividad y el factor clave de la adaptación corporativa por tres razones: son dificiles de acumular, susceptibles de usos simultáneos y constituyen inputs y outputs de las actividades empresariales" (VILLAFAÑE, 2000: 36).

Entre los valores intangibles que Peters y Waterman identificaron, destacan algunas de las principales áreas de intervención de los programas de comunicación estratégica, por ejemplo, la identidad y la cultura organizacional. Los hallazgos de Peters y Waterman definitivamente contribuyeron a ratificar la importancia de la comunicación estratégica en la gestión empresarial. De acuerdo con Villafañe:

"la gestión empresarial no es un valor absoluto en si mismo, sino que está poderosamente influida por valores sociales y culturales; que los directivos son, además de gestores, fabricantes de significados y de simbolos y que el mito y los rituales deben ser introducidos en el management porque sus productos y/o servicios deben poseer significados" (VILLAFAÑE, 2000: 36).

En 1999, Joan Costa, destacado comunicólogo español, advirtió el agotamiento de la racionalidad administrativa que descansa básicamente en el management, pronunciándose por el advenimiento de una nueva racionalidad administrativa centrada en la gestión del conocimiento:

"En los últimos cien años no han cesado de desarrollarse modelos de management centrados en la producción y esforzándose por adaptarse a las circunstancias cambiantes. Por el contrario, hoy nuestras empresas todavía no disponen de modelos avanzados para una cultura que ya no es de la producción, sino de las relaciones, el conocimiento y la gestión eficaz de las Comunicaciones y la información" (COSTA, 1999: 13).

La relevancia de la comunicación estratégica en la nueva gestión empresarial ya no está en duda, aún cuando muchas empresas no reparan en la importancia de la efectiva gestión de su conocimiento y en su expresividad. Respecto a la importancia de la 
Comunicación, Joan Costa (1999) sostiene que ésta representa la principal actividad de la empresa, y la proyecta como fundamento de un nuevo paradigma en la gestión empresarial:

"La regla de oro se sintetiza, pues, en este principio: la Comunicación es acción y la acción es Comunicación. Ambas son inseparables y constituyen un todo inextricable. Esta mentalidad es revolucionaria: supone el abandono de los razonamientos nacidos con el industrialismo - ya obsoletos, pero todavía persistentes en muchas empresas-que se empeñaron en la división del trabajo, los procesos y la empresa misma en detrimento de su unidad esencial. Ahora es la Comunicación la que se impone con el vector estratégico y el sistema nervioso central de las organizaciones [...]. Desde el siglo XIX, las empresas funcionaban sobre cuatro pivotes: economía, organización, producción y administración. Hoy, en los umbrales del siglo XXI, el nuevo paradigma tiene cuatro epicentros. Se llaman identidad, cultura, acción y Comunicación” (COSTA, 1999: 15).

En años recientes la complejidad del entorno expresivo y representacional de marcas y organizaciones parece haber desbordado las capacidades de las agencias de publicidad. La retórica publicitaria ha venido registrando la sensible disminución de su efectividad persuasiva. Al exagerar sistemáticamente los atributos de todo tipo de bienes y servicios, la publicidad efectivamente consiguió ventas, pero en el camino fue perdiendo significado, credibilidad y valor. Los excesos de no pocos publicistas naturalmente contribuyeron a devaluar mucha de la credibilidad informativa del discurso publicitario. Las agencias de publicidad han perdido el monopolio que antes ejercían en el desarrollo de la imagen de marca de productos y organizaciones. El prestigio y la reputación de cualquier marca hoy depende menos del esfuerzo publicitario.

Dada la evidente crisis de credibilidad que hoy enfrenta la persuasión publicitaria, celebridades como Al Ries han pretendido reivindicar la importancia de las Relaciones Públicas. Las tesis contenidas en el libro The Fall of Advertising and the Rise of $P R^{6}-\mathrm{el}$ cual Al Ries escribió con su hija Laura - inevitablemente le distanciaron de Jack Trout, su antiguo socio y amigo. En respuesta a Ries, Jack Trout publicó en la revista Advertising Age el ensayo En defensa de la publicidad.

A pesar de las cualidades que Ries advierte en las Relaciones Públicas, la imagen social de esta disciplina sigue siendo objeto de amplios cuestionamientos. En algunos países sencillamente se les considera actividad superficial y gasto accesorio, que a menudo suele ser asociado con expresiones frívolas y poco relevantes para la efectiva generación de valor para la organización. 


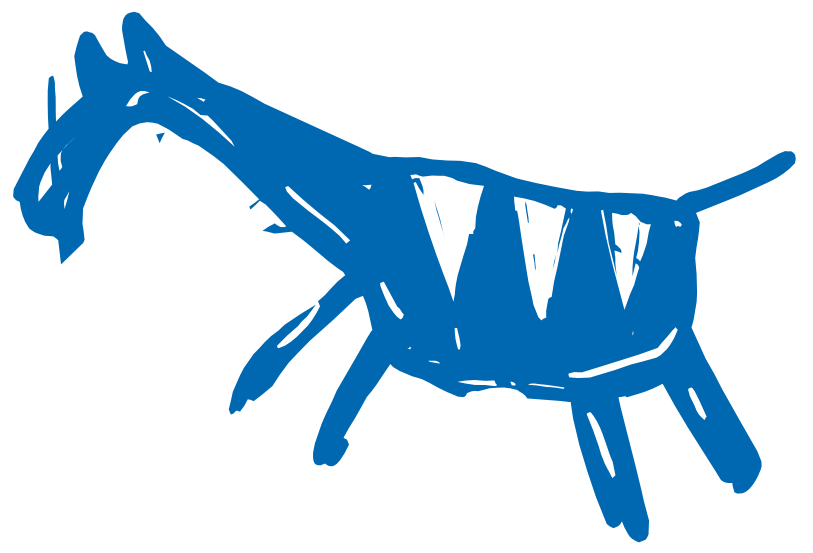

En España, por ejemplo, el destacado investigador Jordi Xifra refiere que en diciembre de 2002 la Asociación de Empresas Consultoras en Relaciones Públicas y Comunicación (Adecec) realizó el estudio La Comunicación y Relaciones Públicas en España. Una de las conclusiones del referido estudio provocó enorme preocupación entre los profesionales de las Relaciones Públicas:

"la ambigüedad de la mayoría de las definiciones de Relaciones Públicas dadas por los profesionales es tal, que podría aplicarse a cualquier otra disciplina comunicativa como, por ejemplo, la publicidad. Esto no hace más que corroborar que si algo caracteri$z a$ a las Relaciones Públicas a la hora de intentar definirlas, es el confucionismo existente acerca de lo que son [...]. Ni desde el campo doctrinal ni desde el profesional nos ha llegado todavía una respuesta razonada. Lo cierto, sin embargo, es que nadie puede negar que el descrédito impera cuando formulamos al ciudadano de a pie que entiende por Relaciones Públicas (si es que entiende algo). Incluso las consultoras que participaron en el estudio de Adecec no sólo consideran que el concepto de Relaciones Públicas está desprestigiado sino que además consideran que está integrado-o desintegrado, según como se mire - en el de Comunicación" (XIFRA, 2003: XVII).

Frente a la visión inmediatista y fragmentaria de la agencia de publicidad, cuya eficacia persuasiva básicamente repara en el impacto sobre potenciales consumidores, y el sensible desprestigio que enfrentan las Relaciones Públicas, la comunicación estratégica reivindica la necesidad de recuperar la confianza del mayor número posible de públicos, afirmando que el valor de la acción-comunicación (COSTA, 1999), reside en la congruencia que admite la expresividad de las acciones comunicativas emprendidas, y para ello es indispensable apostar a la consistencia y al tiempo.

De acuerdo con Charles Fombrun y Cees Van Riel (2003), toda reputación positiva suele estar asociada a cinco atributos empresariales: visibilidad, diferenciación, autenticidad, transparencia y fortaleza. La efectiva gestión de intangibles representa el mejor contra irritante (McLuhan) contra la indiferenciación derivada de los excesos retóricos de la publicidad y la notoria homogeneidad de la oferta. Frente a la visión inmediatista y fragmentaria de la agencia de publicidad, cuya eficacia persuasiva básicamente repara en el impacto sobre potenciales consumidores, la comunicación estratégica reivindica la necesidad de recuperar la confianza del mayor número posible de públicos, afirmando que el valor de la acción-comunicación (COSTA, 1999) reside 
en la congruencia de las acciones comunicativas emprendidas y, para ello, es indispensable apostar a la consistencia y al tiempo.

Los clientes hoy demandan mejor amortización de sus inversiones en materia de Comunicaciones. La campaña publicitaria debe estar subordinada al programa integral de comunicaciones estratégicas, el cual naturalmente responde a las exigencias impuestas por el proyecto empresarial, que no necesariamente debemos suponer como explícito. Las posibilidades de establecer una auténtica diferenciación de identidades de marca subyacen en el imaginario de los intangibles. No es posible improvisar intangibles ni copiarlos con facilidad. La identificación emocional es clave para asegurar la fidelidad de las audiencias meta.

\section{La contribución del pensamiento de Marshall McLuhan en el desarrollo de un enfoque integral de comunicaciones estratégicas}

Ante el desencantamiento de la publicidad y las Relaciones Públicas, prestadores de nuevos servicios de Comunicación han intentado abrir mercados profesionales emergentes, aprovechando las evidentes insuficiencias de las agencias de publicidad y el descrédito que todavía enfrentan los profesionales de las Relaciones Públicas en muchos países. Entre los nuevos servicios especializados de Comunicación destaca el cabildeo, marketing electoral, media training, la consultoría en imagen pública, la comunicación ante situaciones de crisis, la comunicación con públicos financieros, la comunicación filantrópica etc.

Es un grave error suponer que alguno de los servicios emergentes representa la solución a las necesidades de expresividad y desarrollo de imagen de marca de cualquier institución, como suelen afirmar algunos consultores. Los servicios antes referidos pueden ser incorporados al mix de Comunicación Institucional estratégica - siempre y cuando las necesidades de expresividad institucional efectivamente lo justifiquen. De acuerdo con Joan Costa:

"La auténtica solución a estos requerimientos es exactamente un nuevo planteamiento estratégico e integrador para la gestión de las Comunicaciones. Un nuevo enfoque integral que no cae en el error de seguir en la clásica separación de la Comunicación y la acción, sino que se implica y se imbrica en la línea estratégica de acción y management de la empresa" (COSTA, 1999: 14).

Marshall McLuhan recomendó observar un enfoque eminentemente integral al emprender el análisis de los medios de comunicación: "ya que ningún medio tiene sentido o existe a solas, sino solamente en interacción constante con otros medios" (McLUHAN, 1996: 46). Con base en la ecología de medios y a partir de algunas de las tesis de Marshall McLuhan para distinguir entre medios fríos y calientes, es posible de- 
LA POSIBLE CONTRIBUCIÓN DE MARSHALL MCLUHAN Y LA ECOLOGÍA DE LOS MEDIOS AL DESARROLLO Y COMPRENSIÓN DE LAS COMUNICACIONES ESTRATÉGICAS • OCTAVIO ISLAS

rivar un innovador enfoque integral de intervención comunicativa que "no incurra en el error de seguir en la clásica separación de la Comunicación y la acción, y que se implique y se imbrica en la línea estratégica de acción y management de la empresa”.

En el segundo capítulo de Comprender a los medios, Marshall McLuhan aborda el tema de los medios fríos y calientes. La definición de los datos que pueden ser transmitidos a través de algún medio de comunicación y el grado de participación de las audiencias para compensar las insuficiencias informativas del medio, son los criterios fundamentales que permiten distinguir los medios fríos de los calientes:

"Alta definición es el estado del ser bien abastecido de datos. Visualmente, una fotografia es una alta definición. Una caricatura es una definición baja por la sencilla razón de que proporciona muy poca información visual. El teléfono es un medio frio o un medio de definición baja debido a que se da al oído una cantidad mezquina de información, y el habla es un medio frio de definición baja, debido a que es muy poco lo que se da y mucho lo que el oyente tiene que completar [...]; los medios cálidos son de poca o baja participación, mientras que los medios fríos son de alta participación para que el público los complete" (McLUHAN, 1996: 47).

Marshall McLuhan decidió desplazar sus conceptos frío y caliente más allá del ámbito específico de los medios de comunicación, al afirmar que los países atrasados son fríos y los países desarrollados calientes: "En términos de medios fríos y calientes, los países atrasados son fríos y nosotros, calientes. El urbanita es caliente y el rústico, frío (McLUHAN, 1996: 47). Marshall McLuhan además reparó en la posibilidad de analizar las expresiones artísticas y culturales con base en los conceptos frío y caliente:

"El vals era un baile mecánico, rápido y caliente, adecuado para la época industrial y su estado animico de pompa y circunstancias. En cambio el twist es una clase de gesticulación improvisada, fría y comprometida. El jazz de la época de los nuevos medios calientes del cine y de la radio era jazz caliente. Sin embargo, el jazz en sí tiende a ser una forma de baile casual y dialogal que carece de las formas mecánicas y repetitivas del vals. El jazz frío apareció muy naturalmente una vez quedó asimilado el primer impacto del cine y de la radio" (McLUHAN, 1996: 47).

Lewis Mumford, refiere McLuhan, también recurrió a la distinción de lo frío y caliente para emprender el análisis de la cuestión urbana:

"A lo largo de The City in History, Lewis Mumford prefiere las ciudades frías, con una estructura más dispersa, a las calientes, muy compactas. Para él, el mejor período de Atenas fue cuando aún prevalecían la mayoría de los hábitos democráticos de participación, propios de la vida aldeana. Entonces pudo manifestarse toda la variedad de la expresión humana de un modo que luego resultó imposible en los centros urbanos altamente desarrollados. Una situación altamente desarrollada es, por definición, pobre 
LA POSIBLE CONTRIBUCIÓN DE MARSHALL MCLUHAN Y LA ECOLOGÍA DE LOS MEDIOS AL DESARROLLO Y COMPRENSIÓN DE LAS COMUNICACIONES ESTRATÉGICAS • OCTAVIO ISLAS

en oportunidades para la participación y rigurosa en sus exigencias de fragmentación a aquellos susceptibles de controlarla" (McLUHAN, 1996: 48).

También en el capítulo Medios calientes y medios fríos de Comprender a los medios, McLuhan desliza la posibilidad de emplear las categorías de frío y caliente para aplicarlas en estudios de imagen pública:

"En The Fourth Branch of Government, Douglas Carter cuenta cómo el personal de las oficinas de prensa de Washington se deleitaba completando o rellenando los blancos en la personalidad de Calvin Coolidge. Como se parecía tanto a una caricatura, se sentían tentados a completar su imagen para él y para su público. Es instructivo que la prensa lo calificara de frío. En el sentido preciso de un medio frí, Coolidge carecía hasta el punto de articulación de datos en su imagen pública que sólo había una palabra para él: era realmente frío" (McLUHAN, 1996: 49).

Con base en todo lo anterior, resulta factible aplicar los conceptos de frío y caliente en estudios de cultura organizacional. Las instituciones calientes permiten menos participación que las frías. Las instituciones calientes excluyen mientras las frías incluyen. El ejército y la iglesia representan estupendos ejemplos de instituciones históricas calientes. En consecuencia, la planeación de las comunicaciones estratégicas para tales organizaciones debe reparar en la selección de medios idóneos para asegurar la deseable homeostasis ecológica. Los medios de comunicación y las tecnologías, en general, intervienen decisivamente en la aceleración de la temperatura de las sociedades como también de las instituciones. En toda institución es posible experimentar el impacto perturbador que produce el transitar de una cultura institucional fría a una caliente. En ocasiones el estilo personal de los directivos o los recursos tecnológicos de la organización pueden atenuar o agudizar el impacto que produce el brusco cambio de temperatura.

En cualquier medio o estructura, señala Marshall McLuhan, hay un punto de ruptura en el cual el sistema puede ser transformado y convertirse en otro. Los medios de comunicación y las tecnologías, en general, juegan un papel protagónico en la dialéctica de los sistemas e instituciones. En las instituciones, los programas de Comunicación Institucional y, lógicamente los medios institucionales, deben estar perfectamente alineados con la naturaleza misma de la institución, sea ésta caliente o fría. La inadecuada selección de medios institucionales puede introducir graves conflictos en las organizaciones. Por ejemplo, los medios especializados de intercambio e información, advierte McLuhan, pueden fragmentar una estructura tribal. Respecto a la pertinente elección de medios en determinadas culturas - ejemplo que efectivamente puede extenderse a las culturas organizacionales -, Marshall McLuhan afirma:

"Hay una gran diferencia si un medio caliente se emplea en una cultura caliente o en una cultura fría. El medio caliente de la radio empleado en una cultura no alfabetizada tiene un violento efecto, muy distinto del que causaría en Inglaterra o América del 
Norte, por ejemplo, donde la radio se concibe como espectáculo. Una cultura fría o con un bajo nivel de alfabetización no puede tomar como espectáculo los medios calientes del cine o de la radio" (McLUHAN, 1996: 50).

Marshall McLuhan incorpora la figura del censor para evitar y / o anunciar oportunamente el cambio en la temperatura. De acuerdo con McLuhan, el censor: "protege nuestro sistema central de valores y nuestro sistema físico con sólo enfriar considerablemente la manifestación de una experiencia” (McLUHAN, 1997: 44). En las organizaciones, los programas integrales de comunicación estratégica precisamente cumplen el papel de censores que propician, evitar y/o anuncian oportunamente los cambios de temperatura.

\section{Conclusión}

Si bien Marshall McLuhan hoy es mundialmente reconocido como el visionario comunicólogo canadiense que anticipó el formidable boom de las comunicaciones digitales, es necesario comprender el pensamiento de McLuhan como holístico y trascender el injusto encasillamiento que suele confinar a las tesis de McLuhan al estricto tema de las tecnologías de Comunicación. Las tesis de McLuhan sirven para comprender en su totalidad la complejidad de las acciones comunicativas, ya sea a través de los efectos que introducen los medios de comunicación en las sociedades como también el pertinente empleo de los medios institucionales en las organizaciones.

Estoy convencido de que el pensamiento de Marshall McLuhan comprende un conjunto de novedosas y provocativas teorías, susceptibles de enriquecer y renovar el congestionado imaginario conceptual de las Comunicaciones Institucionales estratégicas. Desafortunadamente pocos académicos e investigadores han reparado en la posibilidad de recuperar el pensamiento de McLuhan para emprender el análisis y la planeación de Comunicaciones Institucionales estratégicas. Nunca es tarde para rectificar. La compleja y generosa obra del canadiense nos depara aún una gran cantidad de sorpresas.

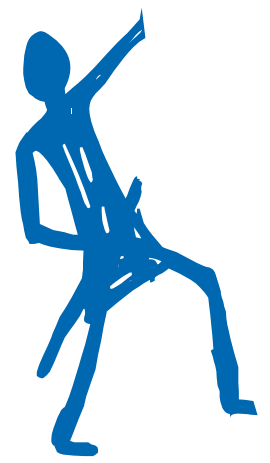


LA POSIBLE CONTRIBUCIÓN DE MARSHALL MCLUHAN Y LA ECOLOGÍA DE LOS MEDIOS AL DESARROLLO Y COMPRENSIÓN DE LAS COMUNICACIONES ESTRATÉGICAS • OCTAVIO ISLAS

\section{Bibliografía}

ÁLVAREZ, T., CABALLERO, M. Vendedores de imagen. Los retos de los nuevos gabinetes de comunicación. España: Paidós, 1998.

Asociación de Empresas Consultoras en Relaciones Públicas y Comunicación (ADECEC). La Comunicación y Relaciones Públicas en España. Radiografía de un sector. Madrid: Pirámide, 2002.

BARQUERO, J. Comunicación y Relaciones Públicas. Madrid: McGraw-Hill, 2001.

BERNAYS, E. The engineering of consent. Oklahoma: University of Oklahoma Press, 1955.

BERRY, J. The interest group society. New York: Longman, 1997.

BONILLA, C. Relaciones Públicas. Factor de competitividad para empresas e instituciones. México: CECSA, 2002.

BUSTAMANTE, E. (Coord.). Hacia un nuevo sistema mundial de comunicación. Las industrias culturales en la era digital. España: Gedisa, 2003.

CAPRIOTTI, P. Planificación estratégica de la imagen corporativa. Barcelona: Ariel, 1999.

CAREY, J. Communication as Culture: Essays on Media and Society. Boston: Unwin Imán, 1989.

COSTA, J. La imagen de empresa. Métodos de comunicación integral. Biblioteca de la Comunicación del CIAC. Madrid: Ibérico Europea de Ediciones, 1977.

. Imagen global. Evolución del diseño de identidad. Barcelona: Ediciones CEAC, 1987.

. La imagen pública. Una ingeniería social. Madrid: Fundesco, 1992.

. La comunicación en acción. Informe sobre la nueva cultura. Madrid: Paidós, 1999.

DE KERCKHOVE, D. The skin of culture: Investigating the new electronic reality. Toronto: Sommerville, 1995.

. Connected intelligence: The arrival of the web society. Toronto: Sommerville, 1997.

. The architecture of intelligence. Boston: Birkhauser, 2001.

DEL PULGAR, L. Comunicación de empresas en entornos turbulentos: Gestión de riesgos, reducción de las incertidumbres y supervivencia. Madrid: ESIC, 1999.

ELÍAS, J., MASCARAY, J.: Más allá de la comunicación interna: la intracomunicación. Barcelona: Gestión 2000, 1998.

ELLUL, J. The Technological Society. New York: Knopf, 1964.

FOMBRUM, C. Reputation: realizing value from the corporate image. Boston: Harvard Business School Press, 1998.

, VAN RIEL, C. Fame \& fortune. How successful companies build winning reputations. Upper Saddle River: Prentice Hall, 2003. 
LA POSIBLE CONTRIBUCIÓN DE MARSHALL MCLUHAN Y LA ECOLOGÍA DE LOS MEDIOS AL DESARROLLO Y COMPRENSIÓN DE LAS COMUNICACIONES ESTRATÉGICAS • OCTAVIO ISLAS

GARBETT, T. Imagen corporativa. Cómo crearla y proyectarla. Colombia: Legis, 1991.

GATES, B. The Road Ahead. U.S.A: Warner Books, 1995.

Business@ @ the speed of though. Using a digital nervous system. U.S.A: Warner Books, 2000.

IND, N. La imagen corporativa. Estrategias para desarrollar programas de identidad eficientes. Madrid: Díaz de Santos, 1997.

INNIS, H. Empire and Communications. Oxford: Clarendon Press, 1950.

The Bias of Communication. Toronto: University of Toronto Press, 1951.

JOHNSON, S. Interface culture: How new technology transforms the way we create and communicate. New York: Harper Collins, 1997.

KOTLER, P. Marketing Management: analysis, planning and Control. New Jersey: Prentice Hall, 1984.

LESSEN, R. Gestión de la cultura corporativa. España: Editorial Díaz de Santos, 1990.

LEVINSON, P. The Soft Edge: A natural history and future of the information revolution. London and New York: Routledge, 1997.

Digital McLuhan. A guide to the information millennium. London-New York: Routledge, 1999.

MAN, C. The intellectual roots of media ecology. En The New Jersey Journal of Communication. Vol. 8. Número 1. Primavera de 2000, p.1-8.

MEYROWITZ, J. No sense of place: the impact of electronic media on social behavior. New York: Oxford University Press, 1985.

McLUHAN, M. The Gutenberg galaxy: The making of typographic man. New York: Mentor, 1962.

\& McLUHAN, E. Laws of Media: The New Science. Toronto: University of Toronto Press, 1988.

. Comprender a los medios. Las extensiones del hombre. Barcelona: Piados, 1996.

MOLES, A., COSTA, J. Publicidad y diseño. El nuevo reto de la comunicación. Buenos Aires, Ediciones Infinito, 1999.

PETERS, T., WATERMAN, R. In Search of Excellence. Cambridge: Harper \& Row, 1981.

PORTER, M. Competitive strategy: techniques for analyzing industries and competitors. New York: The Free Press, 1980.

. Competitive advantage: creating and sustaining superior performance. New York: The Free Press, 1985.

POSTMAN, N. Amusing ourselves to death: public discourse in the age of show business. New York: Viking, 1985. Technopoly: The surrender of culture to technology. New York: Knopf, 1992.

66 - organicom • ANO 2 - NÚMERO $3 \cdot 2^{\circ}$ SEMESTRE DE 2005 
LA POSIBLE CONTRIBUCIÓN DE MARSHALL MCLUHAN Y LA ECOLOGÍA DE LOS MEDIOS AL DESARROLLO Y COMPRENSIÓN DE LAS COMUNICACIONES ESTRATÉGICAS • OCTAVIO ISLAS

, WEINGARTNER, C. Teaching as a subversive activity. New York: Delacorte, 1969.

RIES, A., TROUT, J. Positioning. The battle for your mind. U.S.A: HarperBusiness, 1994.

RIES, L. The fall of advertising and the rise of the PR. U.S.A: HarperCollins, 1994.

SCHEINSOHN, D. Más allá de la imagen corporativa. Cómo crear valor a través de la comunicación estratégica. Buenos Aires: Ediciones Macchi, 1997.

STRATE, L. Communication and cyberspace. Social interaction in an electronic environment. New Jersey: Hampton Press, 1997.

TAPSCOTT, D., TICOLL, D., LOWY, A. Capital digital. El poder de las redes de negocios. España: Taurus, 2001.

VAN RIEL, C. Comunicación corporativa. España: Prentice Hall, 1997.

Identiteit en imago. Recente inzichten in corporate communication, theorie \& praktijk. Rótterdam: Academic Service, 2003.

VILLAFAÑE, J. La gestión profesional de la imagen corporativa. Madrid: Ediciones Pirámide, 1999. Imagen positiva. Gestión estratégica de la imagen de las empresas. Madrid: Ediciones Pirámide, 2000. . La Buena reputación. Claves del valor intangible de las empresas. Madrid: Ediciones Pirámide, 2004.

XIFRA, J. Lobbying. Barcelona: Gestión 2000, 1998.

Teoría y estructura de las Relaciones Públicas.

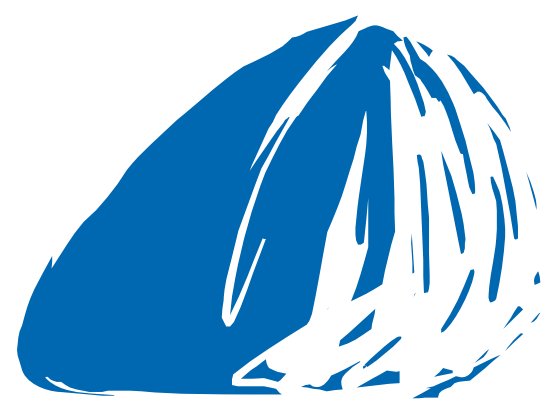

ANO 2 • NÚMERO $3 \cdot 2^{\circ}$ SEMESTRE DE 2005 • organicom • 67 